\title{
Enumeration of points, lines, planes, etc.
}

\author{
by \\ JunE HuH \\ Institute for Advanced Study \\ Princeton, NJ, U.S.A. \\ and \\ Korea Institute for Advanced Study \\ Seoul, Korea \\ BOTONG WANG \\ University of Wisconsin-Madison \\ Madison, WI, U.S.A.
}

\section{Introduction}

One of the earliest results in enumerative combinatorial geometry is the following theorem of de Bruijn and Erdős [dBE]:

Every finite set of points $E$ in a projective plane determines at least $|E|$ lines, unless $E$ is contained in a line.

In other words, if $E$ is not contained in a line, then the number of lines in the plane containing at least two points in $E$ is at least $|E|$. See [dW1] and [dW2] for an interesting account of its history and a survey of known proofs.

The following more general statement, conjectured by Motzkin in [Mo1], was subsequently proved by many in various settings:

Every finite set of points $E$ in a projective space determines at least $|E|$ hyperplanes, unless $E$ is contained in a hyperplane.

Motzkin proved the above for $E$ in real projective spaces [Mo2]. Basterfield and Kelly $[\mathrm{BK}]$ showed the statement in general, and Greene [G] strengthened the result by showing that there is a matching from $E$ to the set of hyperplanes determined by $E$, unless $E$ is contained in a hyperplane:

For every point in $E$ one can choose a hyperplane containing the point in such a way that no hyperplane is chosen twice.

Mason [Ma] and Heron [He] obtained similar results by different methods.

Let $\mathbb{P}$ be the projectivization of an $r$-dimensional vector space over a field, $E \subseteq \mathbb{P}$ be a finite subset not contained in any hyperplane, and $\mathscr{L}$ be the poset of subspaces of $\mathbb{P}$ 
spanned by the subsets of $E$. The poset $\mathscr{L}$ is a graded lattice, and its rank function satisfies the submodular inequality

$$
\operatorname{rank}\left(F_{1}\right)+\operatorname{rank}\left(F_{2}\right) \geqslant \operatorname{rank}\left(F_{1} \vee F_{2}\right)+\operatorname{rank}\left(F_{1} \wedge F_{2}\right) \text { for all } F_{1}, F_{2} \in \mathscr{L} \text {. }
$$

For a non-negative integer $p$, we write $\mathscr{L}^{p}$ for the set of rank-p elements in the lattice $\mathscr{L}$. Thus $\mathscr{L}^{1}$ is the set of points in $E, \mathscr{L}^{2}$ is the set of lines joining points in $E$, and $\mathscr{L}^{r}$ is the set with one element, $\mathbb{P}$. Graded posets obtained in this way are standard examples of geometric lattices $[\mathrm{W}]$. These include the lattice of all subsets of a finite set (boolean lattices), the lattice of all partitions of a finite set (partition lattices), and the lattice of all subspaces of a finite vector space (projective geometries). In [DW2], Dowling and Wilson further generalized the above results for geometric lattices:

For every non-negative integer $p$ less than $\frac{1}{2} r$, there is a matching from the set of rank-at-most-p elements of $\mathscr{L}$ to the set of corank-at-most-p elements of $\mathscr{L}$.

The matching can be chosen to match the minimum of $\mathscr{L}$ to the maximum of $\mathscr{L}$, and hence the above statement covers all the results introduced above. Kung gave another proof of the same result from the point of view of Radon transformations in [Ku1] and [Ku2].

In [DW1] and [DW2], Dowling and Wilson stated the following "top-heavy" conjecture.

Conjecture 1. Let $\mathscr{L}$ be a geometric lattice of rank $r$.

(1) For every non-negative integer $p$ less than $\frac{1}{2} r$,

$$
\left|\mathscr{L}^{p}\right| \leqslant\left|\mathscr{L}^{r-p}\right| .
$$

In fact, there is an injective map $\iota: \mathscr{L}^{p} \rightarrow \mathscr{L}^{r-p}$ satisfying $x \leqslant \iota(x)$ for all $x$.

(2) For every non-negative integer $p$ less than $\frac{1}{2} r$,

$$
\left|\mathscr{L}^{p}\right| \leqslant\left|\mathscr{L}^{p+1}\right| .
$$

In fact, there is an injective map $\iota: \mathscr{L}^{p} \rightarrow \mathscr{L}^{p+1}$ satisfying $x \leqslant \iota(x)$ for all $x$.

The conjecture was reproduced in [St12, Exercise 3.37] and [KRY, Exercise 3.5.7]. For an overview and related results, see [A]. When $\mathscr{L}$ is a boolean lattice or a projective geometry, the validity of Conjecture 1 is a classical result. We refer to $\left[\mathrm{HM}^{+}\right]$and [Stl3] for recent expositions. In these cases, Conjecture 1 implies that $\mathscr{L}$ has the Sperner property:

The maximal number of incomparable elements in $\mathscr{L}$ is the maximum of $\left|\mathscr{L}^{p}\right|$ over $p$. 
Kung proved the second part of Conjecture 1 for partition lattices in [Ku3]. Later he showed the second part of Conjecture 1 for $p \leqslant 2$ when every line contains the same number of points [Ku4].

We now state our main result. As before, we write $\mathbb{P}$ for the projectivization of an $r$-dimensional vector space over a field.

THEOREM 2. Let $E \subseteq \mathbb{P}$ be a finite subset not contained in any hyperplane, and $\mathscr{L}$ be the poset of subspaces of $\mathbb{P}$ spanned by subsets of $E$.

(1) For all non-negative integers $p \leqslant q$ satisfying $p+q \leqslant r$,

$$
\left|\mathscr{L}^{p}\right| \leqslant\left|\mathscr{L}^{r-q}\right|
$$

In fact, there is an injective map $\iota: \mathscr{L}^{p} \rightarrow \mathscr{L}^{r-q}$ satisfying $x \leqslant \iota(x)$ for all $x$.

(2) For every positive integer $p$ less than $\frac{1}{2} r$,

$$
0 \leqslant\left|\mathscr{L}^{p+1}\right|-\left|\mathscr{L}^{p}\right| \leqslant\left(\left|\mathscr{L}^{p}\right|-\left|\mathscr{L}^{p-1}\right|\right)^{\langle p\rangle}
$$

Equivalently, $\left(\left|\mathscr{L}^{0}\right|,\left|\mathscr{L}^{1}\right|-\left|\mathscr{L}^{0}\right|, \ldots,\left|\mathscr{L}^{p+1}\right|-\left|\mathscr{L}^{p}\right|\right)$ is the $h$-vector of a shellable simplicial complex.

The first part of Theorem 2 settles Conjecture 1 for all $\mathscr{L}$ realizable over some field. We believe this to be a good demonstration of the power of the main ingredient in the proof, the decomposition theorem package for intersection complexes [BBD].

The expression $n^{\langle p\rangle}$ used in the second part of Theorems 2 is defined as follows: For any positive integer $p$ and non-negative integer $n$, write $n_{1}, \ldots, n_{p}$ for the unique increasing sequence of non-negative integers satisfying

$$
n=\sum_{k=1}^{p}\left(\begin{array}{c}
n_{k} \\
k
\end{array}\right) .
$$

Then, by definition,

$$
n^{\langle p\rangle}=\sum_{k=1}^{p}\left(\begin{array}{c}
n_{k}+1 \\
k+1
\end{array}\right) .
$$

For $h$-vectors of shellable simplicial complexes, we refer to [Stl1, Chapter II].

Example 3. Modular geometric lattices, such as boolean lattices or finite projective geometries, satisfies a stronger matching property:

For every $p$, there is an injective or surjective map $\iota: \mathscr{L}^{p} \rightarrow \mathscr{L}^{p+1}$ satisfying $x \leqslant \iota(x)$.

As noted before, this implies that modular geometric lattices have the Sperner property. 
Dilworth and Greene constructed in [DG] a configuration of 21 points in any 10dimensional projective space over a field with the property that there is neither injective nor surjective map

$$
\iota: \mathscr{L}^{6} \longrightarrow \mathscr{L}^{7}, \quad x \leqslant \iota(x) .
$$

Canfield $[\mathrm{C}]$ found such "no-matching" successive rank-level sets as above in partition lattices with sufficiently many elements (exceeding $10^{10^{20}}$ ). These geometric lattices satisfy Conjecture 1 but do not have the Sperner property.

Rota conjectured $[\mathrm{R}],[\mathrm{RH}]$ that the sizes of the rank-level sets of a geometric lattice form a unimodal sequence

$$
\left|\mathscr{L}^{0}\right| \leqslant \ldots \leqslant\left|\mathscr{L}^{p-1}\right| \leqslant\left|\mathscr{L}^{p}\right| \geqslant\left|\mathscr{L}^{p+1}\right| \geqslant \ldots \geqslant\left|\mathscr{L}^{r}\right| \text { for some } p \text {. }
$$

Stronger versions of this conjecture were proposed by Mason [Ma]. The unimodality for the "upper half" remains as an outstanding open problem.

Example 4. Let $\lambda$ be a partition of a positive integer, which we view as a Young diagram [F2]. For example, the partition $(4,2,1)$ of 7 corresponds to the Young diagram

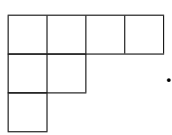

The Young's lattice associated with $\lambda$ is the graded poset $\mathscr{L}_{\lambda}$ of all partitions whose Young diagram fit inside $\lambda$. The poset $\mathscr{L}_{\lambda}$ is usually not a geometric lattice, but Björner and Ekedahl $[\mathrm{BE}]$ showed that $\mathscr{L}_{\lambda}$ satisfies both conclusions of Conjecture 1 when $r$ is the number of boxes in $\lambda$ :

(1) For $p$ less than $\frac{1}{2} r$, there is an injective map $\iota: \mathscr{L}^{p} \rightarrow \mathscr{L}^{r-p}$ satisfying $x \leqslant \iota(x)$ for all $x$.

(2) For $p$ less than $\frac{1}{2} r$, there is an injective map $\iota: \mathscr{L}^{p} \rightarrow \mathscr{L}^{p+1}$ satisfying $x \leqslant \iota(x)$ for all $x$.

However, according to Stanton [Stt], Young's lattice for the partition $(8,8,4,4)$ defines a non-unimodal sequence

$$
\begin{aligned}
& \left(\left|\mathscr{L}_{\lambda}^{0}\right|,\left|\mathscr{L}_{\lambda}^{1}\right|,\left|\mathscr{L}_{\lambda}^{2}\right|, \ldots,\left|\mathscr{L}_{\lambda}^{24}\right|\right) \\
& \quad=(1,1,2,3,5,6,9,11,15,17,21,23,27,28,31,30,31,27,24,18,14,8,5,2,1) .
\end{aligned}
$$

Face lattices of simplicial polytopes behave similarly, starting from dimension 20 [BL], [B]. See $[Z$, Chapter 8$]$ for a discussion of unimodality in the case of polytopes. 


\section{The graded Möbius algebra}

We use the language of matroids, and use $[\mathrm{W}]$ and $[\mathrm{O}]$ as basic references. Let $r$ and $n$ be positive integers, and let $\mathrm{M}$ be a rank- $r$ simple matroid on the ground set

$$
E=\{1, \ldots, n\}
$$

Write $\mathscr{L}$ for the lattice of flats of M. We define a graded analogue of the Möbius algebra for $\mathscr{L}$.

Definition 5. Introduce symbols $y_{F}$, one for each flat $F$ of $\mathrm{M}$, and construct vector spaces

$$
B^{p}(\mathrm{M})=\bigoplus_{F \in \mathscr{L}^{p}} \mathbb{Q} y_{F} \quad \text { and } \quad B^{*}(\mathrm{M})=\bigoplus_{F \in \mathscr{L}} \mathbb{Q} y_{F} .
$$

We equip $B^{*}(\mathrm{M})$ with the structure of a commutative graded algebra over $\mathbb{Q}$ by setting

$$
y_{F_{1}} y_{F_{2}}= \begin{cases}y_{F_{1} \vee F_{2}}, & \text { if } \operatorname{rank}\left(F_{1}\right)+\operatorname{rank}\left(F_{2}\right)=\operatorname{rank}\left(F_{1} \vee F_{2}\right), \\ 0, & \text { if } \operatorname{rank}\left(F_{1}\right)+\operatorname{rank}\left(F_{2}\right)>\operatorname{rank}\left(F_{1} \vee F_{2}\right) .\end{cases}
$$

For simplicity, we write $y_{1}, \ldots, y_{n}$ instead of $y_{\{1\}}, \ldots, y_{\{n\}}$.

Maeno and Numata introduced this algebra in a slightly different form in [MN], and used it to show that modular geometric lattices have the Sperner property. Note that $B^{*}(\mathrm{M})$ is generated by $B^{1}(\mathrm{M})$ as an algebra: If $I_{F}$ is any basis of a flat $F$ of $\mathrm{M}$, then

$$
y_{F}=\prod_{i \in I_{F}} y_{i} .
$$

Unlike its ungraded counterpart, which is isomorphic to the product of $\mathbb{Q}$ 's as a $\mathbb{Q}$-algebra [So], the graded Möbius algebra $B^{*}(\mathrm{M})$ has a non-trivial algebra structure. Define

$$
L=\sum_{i \in E} y_{i}
$$

We deduce Theorem 2 from the following algebraic statement. Similar injectivity properties have appeared in the context of Kac-Moody Schubert varieties [BE] and toric hyperkähler varieties [Ha].

THEOREM 6. For non-negative integer $p$ less than $\frac{1}{2} r$, the multiplication map

$$
\begin{aligned}
B^{p}(\mathrm{M}) & \longrightarrow B^{r-p}(\mathrm{M}), \\
\xi & \longmapsto L^{r-2 p} \xi,
\end{aligned}
$$

is injective, when $\mathrm{M}$ is realizable over some field. 
It follows that, for non-negative integers $p \leqslant q$ satisfying $p+q \leqslant r$, the multiplication map

$$
\begin{aligned}
B^{p}(\mathrm{M}) & \longrightarrow B^{r-q}(\mathrm{M}), \\
\xi & \longmapsto L^{r-p-q} \xi,
\end{aligned}
$$

is injective, when $\mathrm{M}$ is realizable over some field. To deduce the first part of Theorem 2 from this, consider the matrix of the multiplication map with respect to the standard bases of the source and the target. Entries of this matrix are labeled by pairs of elements of $\mathscr{L}$, and all the entries corresponding to incomparable pairs are zero. The matrix has full rank, so there is a maximal square submatrix with non-zero determinant. In the standard expansion of this determinant, there must be a non-zero term, and the permutation corresponding to this term produces the injective map $\iota$. The second part of Theorem 2 also follows from Theorem 6 . To see this, note that the algebra $B^{*}(\mathrm{M})$ is generated by its degree-1 elements, and apply Macaulay's theorem to the quotient of $B^{*}(\mathrm{M})$ by the ideal generated by $L$ [Stl1, Chapter II, Corollary 2.4].

Conjecture 7. Theorem 6 holds without the assumption of realizability.

Let $\mathrm{M}$ be as before, and let $\overline{\mathrm{M}}$ be a simple matroid on the ground set

$$
\bar{E}=\{0,1, \ldots, n\} .
$$

Let $\overline{\mathscr{L}}$ be the lattice of flats of $\overline{\mathrm{M}}$. We suppose that $\mathrm{M}=\overline{\mathrm{M}} / 0$, that is, $\mathrm{M}$ is obtained from $\overline{\mathrm{M}}$ by contracting the element 0 .

Definition 8. Introduce variables $x_{\bar{F}}$, one for each non-empty proper flat $\bar{F}$ of $\overline{\mathrm{M}}$, and set

$$
S_{\overline{\mathrm{M}}}=\mathbb{Q}\left[x_{\bar{F}}\right]_{\bar{F} \neq \varnothing, \bar{F} \neq \bar{E}, \bar{F} \in \overline{\mathscr{L}} .}
$$

The Chow ring $A^{*}(\overline{\mathrm{M}})$ is the quotient of $S_{\overline{\mathrm{M}}}$ by the ideal generated by the linear forms

$$
\sum_{i_{1} \in \bar{F}} x_{\bar{F}}-\sum_{i_{2} \in \bar{F}} x_{\bar{F}}
$$

one for each pair of distinct elements $i_{1}$ and $i_{2}$ of $\bar{E}$, and the quadratic monomials

$$
x_{\bar{F}_{1}} x_{\bar{F}_{2}},
$$

one for each pair of incomparable non-empty proper flats of $\overline{\mathrm{M}}$. 
The algebra $A^{*}(\overline{\mathrm{M}})$ and its generalizations were studied by Feichtner and Yuzvinsky in $[\mathrm{FY}]$. For every $i$ in $E$, we define an element of $A^{1}(\overline{\mathrm{M}})$ by setting

$$
\beta_{i}=\sum_{\bar{F}} x_{\bar{F}},
$$

where the sum is over all flats $\bar{F}$ of $\overline{\mathrm{M}}$ that contain 0 and do not contain $i$. The linear relations show that we may equivalently define

$$
\beta_{i}=\sum_{\bar{F}} x_{\bar{F}}
$$

where the sum is over all flats $\bar{F}$ of $\overline{\mathrm{M}}$ that contain $i$ and do not contain 0 . We record here three basic implications of the defining relations of $A^{*}(\overline{\mathrm{M}})$ :

(R1) When $\bar{F}$ is a non-empty proper flat of $\overline{\mathrm{M}}$ containing exactly one of $i$ and 0 ,

$$
\beta_{i} \cdot x_{\bar{F}}=0
$$

This follows from the quadratic monomial relations.

(R2) For every element $i$ in $E$,

$$
\beta_{i} \cdot \beta_{i}=0 .
$$

This follows from the previous statement.

(R3) For any two maximal chains of non-empty proper flats of $\bar{M}$, say $\left\{\bar{F}_{k}\right\}_{1 \leqslant k}$ and $\left\{\bar{G}_{k}\right\}_{1 \leqslant k}$,

$$
\prod_{k=1}^{r} x_{\bar{F}_{k}}=\prod_{k=1}^{r} x_{\bar{G}_{k}} \neq 0 .
$$

The proofs of (R1) and (R2) are straightforward. The proof of (R3) can be found in $[\mathrm{AHK}, \S 5]$.

Proposition 9. There is a unique injective graded $\mathbb{Q}$-algebra homomorphism

$$
\begin{gathered}
\varphi: B^{*}(\mathrm{M}) \longrightarrow A^{*}(\overline{\mathrm{M}}), \\
y_{i} \longmapsto \beta_{i} .
\end{gathered}
$$

Proof. First, we show that there is a well-defined $\mathbb{Q}$-linear map

$$
\begin{aligned}
\varphi: B^{*}(\mathrm{M}) & \longrightarrow A^{*}(\overline{\mathrm{M}}), \\
y_{F} & \longmapsto \prod_{i \in I_{F}} \beta_{i},
\end{aligned}
$$


where $I_{F}$ is any basis of a flat $F$ of M. In other words, if $J_{F}$ is any other basis of $F$, then

$$
\prod_{i \in I_{F}} \beta_{i}=\prod_{i \in J_{F}} \beta_{i}
$$

Since any basis of $F$ can be obtained from any other basis of $F$ by a sequence of elementary exchanges, it is enough to check the equality in the special case when $I_{F} \backslash J_{F}=\{1\}$ and $J_{F} \backslash I_{F}=\{2\}$. Assuming that this is the case, we write the left-hand side of the claimed equality by

$$
\left(\prod_{i \in I_{F} \cap J_{F}} \beta_{i}\right)\left(\sum_{\bar{G}} x_{\bar{G}}\right)
$$

where the sum is over all non-empty proper flats $\bar{G}$ of $\overline{\mathrm{M}}$ that contain 0 and do not contain 1. The relation (R1) shows that we may take the sum only over those $\bar{G}$ satisfying

$$
0 \in \bar{G}, \quad 1 \notin \bar{G} \text { and } \quad I_{F} \cap J_{F} \subseteq \bar{G} .
$$

Since $I_{F} \cup\{0\}$ and $J_{F} \cup\{0\}$ are bases of the same flat of $\overline{\mathrm{M}}$, the above condition is equivalent to

$$
0 \in \bar{G}, \quad 2 \notin \bar{G} \quad \text { and } \quad I_{F} \cap J_{F} \subseteq \bar{G} .
$$

This proves the claimed equality, which shows that $\varphi$ is a well-defined linear map.

Second, we show that $\varphi$ is a ring homomorphism. Given flats $F_{1}$ and $F_{2}$ of M, we show that

$$
\left(\prod_{i \in I_{F_{1}}} \beta_{i}\right)\left(\prod_{i \in I_{F_{2}}} \beta_{i}\right)=0 \quad \text { when the rank of } F_{1} \vee F_{2} \text { is less than }\left|I_{F_{1}}\right|+\left|I_{F_{2}}\right| .
$$

If the independent sets $I_{F_{1}}$ and $I_{F_{2}}$ intersect, this follows from the relation (R2). If otherwise, the condition on the rank of $F_{1} \vee F_{2}$ implies that there are two distinct bases of $F_{1} \vee F_{2}$ contained in $I_{F_{1}} \cup I_{F_{2}}$, say

$$
I_{F_{1} \vee F_{2}} \subseteq I_{F_{1}} \cup I_{F_{2}} \text { and } J_{F_{1} \vee F_{2}} \subseteq I_{F_{1}} \cup I_{F_{2}} .
$$

Using the first part of the proof, once again from the relation (R2), we deduce that

$$
\begin{aligned}
\left(\prod_{i \in I_{F_{1}}} \beta_{i}\right)\left(\prod_{i \in I_{F_{2}}} \beta_{i}\right) & =\left(\prod_{i \in I_{F_{1} \vee F_{2}}} \beta_{i}\right)\left(\prod_{i \in I_{F_{1}} \cup I_{F_{2}} \backslash I_{F_{1} \vee F_{2}}} \beta_{i}\right) \\
& =\left(\prod_{i \in J_{F_{1} \vee F_{2}}} \beta_{i}\right)\left(\prod_{i \in I_{F_{1}} \cup I_{F_{2}} \backslash I_{F_{1} \vee F_{2}}} \beta_{i}\right)=0 .
\end{aligned}
$$

This completes the proof that $\varphi$ is a ring homomorphism. 
Third, we show that $\varphi$ is injective in degree $r$. Choose any ordered basis $\left\{i_{1}, \ldots, i_{r}\right\}$ of M. For each $q=1, \ldots, r$, we let $\bar{G}_{q}$ be the closure of $\left\{0, i_{1}, \ldots, i_{q-1}\right\}$ in $\overline{\mathrm{M}}$. We deduce from the relation $(\mathrm{R} 1)$ that

$$
\left(\beta_{i_{1}} \ldots \beta_{i_{r-1}}\right) \beta_{i_{r}}=\left(\beta_{i_{1}} \ldots \beta_{i_{r-1}}\right) x_{\bar{G}_{r}} .
$$

Similarly, for any positive integer $q \leqslant r$, we have

$$
\left(\beta_{i_{1}} \ldots \beta_{i_{q-2}} \beta_{i_{q-1}}\right) x_{\bar{G}_{q}}=\left(\beta_{i_{1}} \ldots \beta_{i_{q-2}}\right) x_{\bar{G}_{q-1}} x_{\bar{G}_{q}}=x_{\bar{G}_{1}} \ldots x_{\bar{G}_{q-1}} x_{\bar{G}_{q}}
$$

since $\bar{G}_{q-1}$ is the only flat of $\bar{M}$ containing $\bar{G}_{q-1}$, comparable to $\bar{G}_{q}$, and not containing $i_{q-1}$. Combining the above formulas, we deduce from the relation (R3) that

$$
\beta_{i_{1}} \ldots \beta_{i_{r}}=x_{\bar{G}_{1}} \ldots x_{\bar{G}_{r}} \neq 0
$$

This proves that $\varphi$ is injective in degree $r$.

Last, we show that $\varphi$ is injective in any degree $q$ less than $r$. For this we analyze the bilinear map given by the multiplication

$$
\varphi\left(B^{q}(\mathrm{M})\right) \times \bigoplus_{\bar{G}} \mathbb{Q} x_{\bar{G}} \longrightarrow A^{q+1}(\overline{\mathrm{M}})
$$

where the sum is over all rank- $(q+1)$ flats $\bar{G}$ of $\overline{\mathrm{M}}$ containing 0 . For any independent set $\left\{i_{1}, \ldots, i_{q}\right\}$ of $\mathrm{M}$, we claim that, for any $\bar{G}$ as in the previous sentence,

$$
\left(\beta_{i_{1}} \ldots \beta_{i_{q}}\right) x_{\bar{G}} \neq 0 \text { if and only if } \bar{G} \text { is the closure of }\left\{0, i_{1}, \ldots, i_{q}\right\} \text { in } \overline{\mathrm{M}} \text {. }
$$

The "if" statement follows from the analysis made above. For the "only if" statement, suppose that the product is non-zero. Since $\bar{G}$ contains 0 , it must contain $i_{1}, \ldots, i_{q}$ by the relation (R1). Since $\bar{G}$ and the closure both have the same rank, we have that

$$
\bar{G} \text { is the closure of }\left\{0, i_{1}, \ldots, i_{q}\right\} \text { in } \overline{\mathrm{M}} \text {. }
$$

This proves the claimed equivalence, and it follows that the image of the basis $\left\{y_{F}\right\}$ of $B^{q}(\mathrm{M})$ under $\varphi$ is linearly independent in $A^{q}(\overline{\mathrm{M}})$.

\section{The simplex, the cube, and the permutohedron}

In this section, we give a toric preparation for the proof of our main result, Theorem 6 . For undefined terms in toric geometry and intersection theory, we refer to [F1] and [F3]. All the Chow groups and rings will have rational coefficients. 
As in the previous section, we fix a positive integer $n$ and work with the sets

$$
E=\{1, \ldots, n\} \quad \text { and } \quad \bar{E}=\{0,1, \ldots, n\} .
$$

Let $\mathbb{Z}^{\bar{E}}$ be the abelian group generated by the basis vectors $\mathbf{e}_{i}$ corresponding to $i \in \bar{E}$. For an arbitrary subset $\bar{I} \subseteq \bar{E}$, we define

$$
\mathbf{e}_{\bar{I}}=\sum_{i \in \bar{I}} \mathbf{e}_{i} .
$$

We associate with $\bar{E}$ the abelian group $N_{\bar{E}}=\mathbb{Z}^{\bar{E}} / \mathbb{Z} \mathbf{e}_{\bar{E}}$ and the vector space

$$
N_{\bar{E}, \mathbb{R}}=\mathbb{R}^{\bar{E}} / \mathbb{R} \mathbf{e}_{\bar{E}} .
$$

(1) Let $\Sigma\left(\mathrm{S}_{n}\right) \subseteq N_{\bar{E}, \mathbb{R}}$ be the image of the normal fan of the standard $n$-dimensional simplex

$$
\mathrm{S}_{n}=\operatorname{conv}\left\{\mathbf{e}_{0}, \mathbf{e}_{1}, \ldots, \mathbf{e}_{n}\right\} \subseteq \mathbb{R}^{\bar{E}} .
$$

There are $n+1$ maximal cones in $\Sigma\left(\mathrm{S}_{n}\right)$, one for each maximal proper subset $\bar{I}$ of $\bar{E}$ :

$$
\sigma_{\bar{I}}=\operatorname{cone}\left\{\mathbf{e}_{i}: i \in \bar{I}\right\} \subseteq N_{\bar{E}, \mathbb{R}} .
$$

This fan defines the $n$-dimensional projective space $\mathbb{P}^{n}$, whose homogeneous coordinates are labeled by $i \in \bar{E}$.

(2) Let $\Sigma\left(\mathrm{C}_{n}\right) \subseteq \mathbb{R}^{E}$ be the normal fan of the standard $n$-dimensional cube

$$
\mathrm{C}_{n}=\operatorname{conv}\left\{ \pm \mathbf{e}_{1}, \ldots, \pm \mathbf{e}_{n}\right\} \subseteq \mathbb{R}^{E} .
$$

There are $2^{n}$ maximal cones in $\Sigma\left(\mathrm{C}_{n}\right)$, one for each subset $I$ of $E$ :

$$
\sigma_{I}=\operatorname{cone}\left\{\mathbf{e}_{i}: i \in I\right\}-\operatorname{cone}\left\{\mathbf{e}_{i}: i \notin I\right\} \subseteq \mathbb{R}^{E} .
$$

This fan defines the product of $n$ projective lines $\left(\mathbb{P}^{1}\right)^{n}$, whose multi-homogeneous coordinates are labeled by $i \in E$.

(3) Let $\Sigma\left(\mathrm{P}_{n}\right) \subseteq N_{\bar{E}, \mathbb{R}}$ be the image of the normal fan of the $n$-dimensional permutohedron

$$
\mathrm{P}_{n}=\operatorname{conv}\left\{\left(x_{0}, \ldots, x_{n}\right): x_{0}, \ldots, x_{n} \text { is a permutation of } 0, \ldots, n\right\} \subseteq \mathbb{R}^{\bar{E}} .
$$

There are $(n+1)$ ! maximal cones in $\Sigma\left(\mathrm{P}_{n}\right)$, one for each maximal chain $\mathscr{I}$ in $2^{\bar{E}}$ :

$$
\sigma_{\mathscr{I}}=\operatorname{cone}\left\{\mathbf{e}_{\bar{I}}: \bar{I} \in \mathscr{I}\right\} \subseteq N_{\bar{E}, \mathbb{R}} .
$$

This fan defines the $n$-dimensional permutohedral space, denoted $X_{A_{n}}$. See [BB] for a detailed study of $X_{A_{n}}$ and its analogues for other root systems. 
The inclusion $\mathbb{Z}^{E} \subseteq \mathbb{Z}^{\bar{E}}$ induces an isomorphism

$$
\psi^{-1}: \mathbb{R}^{E} \longrightarrow N_{\bar{E}, \mathbb{R}}
$$

This identifies the underlying vector spaces of the normal fans $\Sigma\left(\mathrm{S}_{n}\right), \Sigma\left(\mathrm{P}_{n}\right)$ and $\Sigma\left(\mathrm{C}_{n}\right)$ :

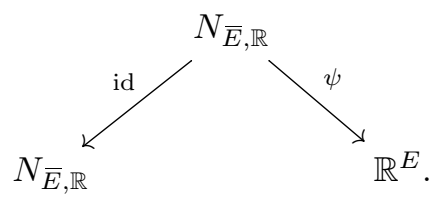

We observe that id and $\psi$ induce morphisms between the fans and their toric varieties:

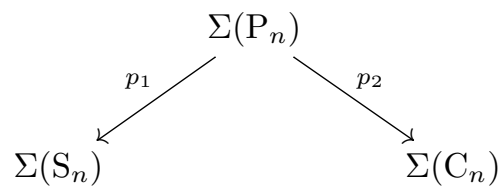

and

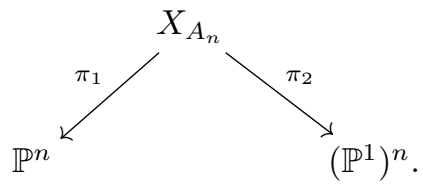

The morphism $p_{1}$ is the standard barycentric subdivision. We check that $p_{2}$ is a subdivision.

Proposition 10. The isomorphism $\psi$ induces a morphism $p_{2}$.

In other words, the image of a cone in $\Sigma\left(\mathrm{P}_{n}\right)$ under $\psi$ is contained in a cone in $\Sigma\left(\mathrm{C}_{n}\right)$.

Proof. For each $i \in E$, define $\psi_{i}$ as the composition of $\psi$ with the $i$ th projection

$$
\psi_{i}=\operatorname{proj}_{i} \circ \psi, \quad \operatorname{proj}_{i}: \mathbb{R}^{E} \longrightarrow \mathbb{R}^{\{i\}} \simeq \mathbb{R} .
$$

For any subset $\bar{I} \subseteq \bar{E}$, we have

$$
\psi_{i}\left(\mathbf{e}_{\bar{I}}\right)= \begin{cases}\mathbf{e}_{i}, & \text { if } \bar{I} \text { contains } i \text { and does not contain } 0, \\ -\mathbf{e}_{i}, & \text { if } \bar{I} \text { contains } 0 \text { and does not contain } i, \\ 0, & \text { otherwise. }\end{cases}
$$

It is enough to check that $\psi_{i}$ induces a morphism $\Sigma\left(\mathrm{P}_{n}\right) \rightarrow \Sigma\left(\mathrm{C}_{1}\right)$.

Recall that any non-zero cone in the normal fan of $\mathrm{P}_{n}$ is of the form

$$
\sigma_{\mathscr{I}}=\operatorname{cone}\left\{\mathbf{e}_{\bar{I}}: \bar{I} \in \mathscr{I}\right\}
$$

where $\mathscr{I}$ is a non-empty chain in $2^{\bar{E}}$. Viewing $\mathscr{I}$ as an ordered collection of sets, we see that $\psi_{i}\left(\sigma_{\mathscr{I}}\right)$ is contained in the cone generated by

$$
\begin{cases}\mathbf{e}_{i}, & \text { if } i \text { appears before } 0 \text { in } \mathscr{I}, \\ -\mathbf{e}_{i}, & \text { if } i \text { appears after } 0 \text { in } \mathscr{I} .\end{cases}
$$

Thus, the image of a cone in $\Sigma\left(\mathrm{P}_{n}\right)$ under $\psi_{i}$ is contained in a cone in $\Sigma\left(\mathrm{C}_{1}\right)$, for each $i \in E$. 
Geometrically, $\pi_{1}$ is the blow-up of all the torus-invariant points in $\mathbb{P}^{n}$, all the strict transforms of torus-invariant $\mathbb{P}^{1}$ s in $\mathbb{P}^{n}$, all the strict transforms of torus-invariant $\mathbb{P}^{2}$ 's in $\mathbb{P}^{n}$, and so on. The map $\pi_{2}$ is the blow-up of points $0^{n}$ and $\infty^{n}$, all the strict transforms of torus-invariant $\mathbb{P}^{1}$ 's in $\left(\mathbb{P}^{1}\right)^{n}$ containing $0^{n}$ or $\infty^{n}$, all the strict transforms of torusinvariant $\left(\mathbb{P}^{1}\right)^{2}$ s in $\left(\mathbb{P}^{1}\right)^{n}$ containing $0^{n}$ or $\infty^{n}$, and so on.

Remark 11. For later use, we record here a combinatorial description of the pull-back of piecewise linear functions under the linear map $\psi_{i}=\operatorname{proj}_{i} \circ \psi$ :

Let $\alpha$ be the piecewise linear function on $\Sigma\left(\mathrm{C}_{1}\right)$ determined by its values

$$
\alpha\left(\mathbf{e}_{i}\right)=1 \quad \text { and } \quad \alpha\left(-\mathbf{e}_{i}\right)=0 .
$$

Then $\psi_{i}^{*}(\alpha)$ is the piecewise linear function on $\Sigma\left(\mathrm{P}_{n}\right)$ determined by its values

$$
\psi_{i}^{*}(\alpha)\left(\mathbf{e}_{\bar{I}}\right)= \begin{cases}1, & \text { if } \bar{I} \text { contains } i \text { and does not contain } 0, \\ 0, & \text { otherwise. }\end{cases}
$$

Using the correspondence between piecewise linear functions on fans and torusinvariant divisors on toric varieties [F1, Chapter 3], the above can be used to describe the pull-back homomorphism between the Chow rings

$$
\pi_{2}^{*}: A^{*}\left(\left(\mathbb{P}^{1}\right)^{n}\right) \longrightarrow A^{*}\left(X_{A_{n}}\right) .
$$

Explicitly, writing $y_{i}$ for the divisor of $\mathbf{e}_{i}$ in $\left(\mathbb{P}^{1}\right)^{n}$ and $x_{\bar{I}}$ for the divisor of $\mathbf{e}_{\bar{I}}$ in $X_{A_{n}}$,

$$
\pi_{2}^{*}\left(y_{i}\right)=\sum_{\bar{I}} x_{\bar{I}}
$$

where the sum is over all subsets $\bar{I} \subseteq \bar{E}$ that contain $i$ and do not contain 0 .

\section{Proof of Theorem 6}

Let $\mathrm{M}$ be a simple matroid on $E$, and let $\overline{\mathrm{M}}$ be a simple matroid on $\bar{E}$ with $\mathrm{M}=\overline{\mathrm{M}} / 0$. For simplicity, we take $\overline{\mathrm{M}}$ to be the direct sum of $\mathrm{M}$ and the rank-1 matroid on $\{0\}$, so that $\mathrm{M}$ and $\overline{\mathrm{M}}$ share the same set of circuits.

Suppose that $\mathrm{M}$ is realizable over some field. Then $\mathrm{M}$ is realizable over some finite field [O, Corollary 6.8.13], and hence over the algebraically closed field $\overline{\mathbb{F}}_{p}$ for some prime number $p$. The matroid $\overline{\mathrm{M}}$ is realizable over the same field, say by a spanning set of vectors

$$
\overline{\mathscr{A}}=\left\{f_{0}, f_{1}, \ldots, f_{n}\right\} \subseteq \overline{\mathbb{F}}_{p}^{r+1}
$$


Dually, the realization $\overline{\mathscr{A}}$ of $\overline{\mathrm{M}}$ corresponds to an injective linear map between projective spaces

$$
i_{\bar{A}}: \mathbb{P}^{r} \longrightarrow \mathbb{P}^{n}, \quad i_{\mathscr{A}}=\left[f_{0}: f_{1}: \ldots: f_{n}\right]
$$

The collection $\mathscr{A}=\left\{f_{1}, \ldots, f_{n}\right\}$ is a realization of the matroid M.

The restriction of the torus-invariant hyperplanes of $\mathbb{P}^{n}$ to $\mathbb{P}^{r}$ defines an arrangement of hyperplanes in $\mathbb{P}^{r}$, which we denote by the same symbol $\overline{\mathscr{A}}$. We use $i_{\mathscr{A}}$ to construct the commutative diagram

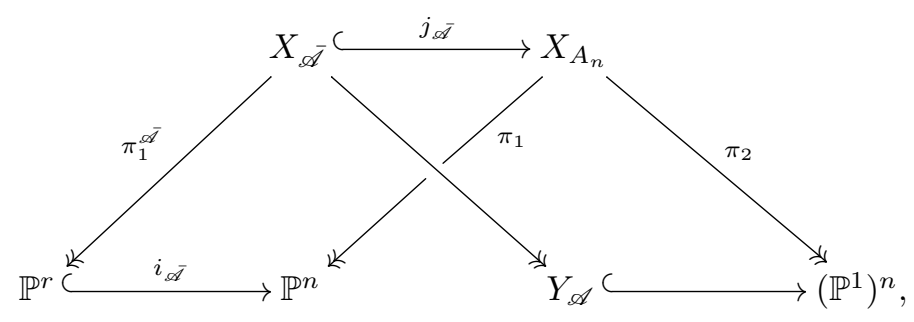

where $X_{\mathscr{A}}$ is the strict transform of $\mathbb{P}^{r}$ under $\pi_{1}$ and $Y_{\mathscr{A}}$ is the image of $X_{\mathscr{A}}$ under $\pi_{2}$.

The induced map $\pi_{1}^{\overline{\mathscr{A}}}$ is the blow-up of all the zero-dimensional flats of $\overline{\mathscr{A}}$, all the strict transforms of 1-dimensional flats of $\overline{\mathscr{A}}$, all the strict transforms of 2-dimensional flats of $\overline{\mathscr{A}}$, and so on. The variety $X_{\mathscr{A}}$ is the wonderful model of $\overline{\mathscr{A}}$ corresponding to the maximal building set [dCP1]. The variety $Y_{\mathscr{A}}$ is studied in [AB], and its affine part centered at $\infty^{n}$ is the reciprocal plane in [EPW], [PS]. To apply the decomposition theorem of $[\mathrm{BBD}]$, we notice that all varieties, maps and sheaves under consideration may be defined over some finite extension of $\mathbb{F}_{p}$.

We know that the Chow ring of $X_{\mathscr{A}}$ is determined by the matroid $\overline{\mathrm{M}}$ : There is an isomorphism of graded algebras

$$
A^{*}(\overline{\mathrm{M}}) \simeq A^{*}\left(X_{\mathscr{A}}\right)
$$

where $x_{\bar{F}}$ is identified with the class of the strict transform of the exceptional divisor produced when blowing up the flat of $\overline{\mathscr{A}}$ corresponding to $\bar{F}$. See [dCP2, 1.1$]$, and also [dCP1] and [FY]. When $\overline{\mathrm{M}}$ is the boolean matroid $\overline{\mathrm{B}}$ on $\bar{E}$, this describes the Chow ring of the permutohedral space $A^{*}\left(X_{A_{n}}\right)$. In general, the pull-back homomorphism

$$
A^{*}(\overline{\mathrm{B}}) \simeq A^{*}\left(X_{A_{n}}\right) \stackrel{j_{\mathscr{A}}^{*}}{\longrightarrow} A^{*}\left(X_{\overline{\mathscr{A}}}\right) \simeq A^{*}(\overline{\mathrm{M}})
$$

is determined by the assignment, for non-empty proper subsets $\bar{I}$ of $\bar{E}$,

$$
x_{\bar{I}} \longmapsto \begin{cases}x_{\bar{I}}, & \text { if } \bar{I} \text { is a flat of } \overline{\mathrm{M}}, \\ 0, & \text { if } \bar{I} \text { is a not flat of } \overline{\mathrm{M}} .\end{cases}
$$


Fix a prime number $\ell$ different from $p$, and consider the $\ell$-adic étale cohomology rings and the $\ell$-adic étale intersection cohomology groups of the varieties in the diagram above. These are $\overline{\mathbb{Q}}_{\ell}$-vector spaces of the form

$$
\mathrm{H}^{*}\left(X, \overline{\mathbb{Q}}_{\ell}\right):=\mathrm{H}^{*}\left(X, \overline{\mathbb{Q}}_{\ell, X}\right) \quad \text { and } \quad \operatorname{IH}^{*}\left(X, \overline{\mathbb{Q}}_{\ell}\right):=\mathrm{H}^{*}\left(X, \mathrm{IC}_{X}\right)
$$

where $\overline{\mathbb{Q}}_{\ell, X}$ and $\mathrm{IC}_{X}$ are constructible complexes of $\overline{\mathbb{Q}}_{\ell}$-sheaves on $X$ as in [BBD]. The blow-up construction of $X_{\mathscr{A}}$ shows that the cycle class map induces an isomorphism of commutative graded $\overline{\mathbb{Q}}_{\ell}$-algebras

$$
A^{*}\left(X_{\mathscr{A}}\right) \otimes_{\mathbb{Q}} \overline{\mathbb{Q}}_{\ell} \simeq \mathrm{H}^{2 *}\left(X_{\mathscr{A}}, \overline{\mathbb{Q}}_{\ell}\right)
$$

see $\left[\mathrm{Ke}\right.$, Appendix]. For the variety $Y_{\mathscr{A}}$, which may be singular, we show in Theorem 14 that there is an isomorphism of commutative graded $\overline{\mathbb{Q}}_{\ell}$-algebras

$$
B^{*}(\mathrm{M}) \otimes_{\mathbb{Q}} \overline{\mathbb{Q}}_{\ell} \simeq \mathrm{H}^{2 *}\left(Y_{\mathscr{A}}, \overline{\mathbb{Q}}_{\ell}\right) .
$$

In general, the intersection cohomology $\operatorname{IH}^{*}\left(X, \overline{\mathbb{Q}}_{\ell}\right)$ is a module over the cohomology $\mathrm{H}^{*}\left(X, \overline{\mathbb{Q}}_{\ell}\right)$, satisfying the Poincaré duality and the hard Lefschetz theorems. See [dCM] for an introduction and precise statements.

We obtain Theorem 6 from the following general observation. Let $f$ be a proper map from an $r$-dimensional smooth projective variety

$$
f: X_{1} \longrightarrow X_{2},
$$

and let $L$ be a fixed ample line bundle on $X_{2}$. Consider the pull-back homomorphism of cohomology in even degrees

$$
\mathrm{H}^{2 *}\left(X_{2}, \overline{\mathbb{Q}}_{\ell}\right) \longrightarrow \mathrm{H}^{2 *}\left(X_{1}, \overline{\mathbb{Q}}_{\ell}\right)
$$

The image of the pull-back is a commutative graded algebra over $\overline{\mathbb{Q}}_{\ell}$, denoted $B^{*}(f)_{\overline{\mathbb{Q}}_{\ell}}$ :

$$
B^{*}(f)_{\overline{\mathbb{Q}}_{\ell}}=\operatorname{im}\left(\mathrm{H}^{2 *}\left(X_{2}, \overline{\mathbb{Q}}_{\ell}\right) \rightarrow \mathrm{H}^{2 *}\left(X_{1}, \overline{\mathbb{Q}}_{\ell}\right)\right) .
$$

$B^{*}(f)_{\overline{\mathbb{Q}} \ell}$ is the cyclic $\mathrm{H}^{2 *}\left(X_{2}, \overline{\mathbb{Q}}_{\ell}\right)$-submodule of $\mathrm{H}^{2 *}\left(X_{1}, \overline{\mathbb{Q}}_{\ell}\right)$ generated by the element 1.

Proposition 12. If $f$ is birational onto its image, then the multiplication map

$$
\begin{aligned}
B^{p}(f)_{\overline{\mathbb{Q}}_{\ell}} & \longrightarrow B^{r-p}(f)_{\overline{\mathbb{Q}}_{\ell}}, \\
\xi & \longmapsto L^{r-2 p} \xi,
\end{aligned}
$$

is injective for every non-negative integer $p$ less than $\frac{1}{2} r$. 
Proof. We reduce to the case when $f$ is surjective. For this consider the factorization

$$
X_{1} \stackrel{g}{\longrightarrow} f\left(X_{1}\right) \stackrel{h}{\longrightarrow} X_{2}, \quad f=h \circ g .
$$

Then $B^{*}(f)_{\overline{\mathbb{Q}}_{\ell}}$ is a subalgebra of $B^{*}(g)_{\overline{\mathbb{Q}}_{\ell}}$, and hence the statement $(f, L)$ follows from $\left(g, h^{*} L\right)$.

Suppose that $f$ is surjective. The decomposition theorem [BBD, $\S 4.3]$ says that the intersection complex of $X_{2}$ appears as a direct summand of the direct image of the constant sheaf $\overline{\mathbb{Q}}_{\ell}$ on $X_{1}$ :

$$
R f_{*} \overline{\mathbb{Q}}_{\ell, X_{1}} \simeq \mathrm{IC}_{X_{2}} \oplus \mathscr{C}
$$

Taking cohomology of both sides, we obtain a splitting injection of $\mathrm{H}^{*}\left(X_{2}, \overline{\mathbb{Q}}_{\ell}\right)$-modules

$$
\Phi: \mathrm{IH}^{*}\left(X_{2}, \overline{\mathbb{Q}}_{\ell}\right) \longrightarrow \mathrm{H}^{*}\left(X_{1}, \overline{\mathbb{Q}}_{\ell}\right)
$$

Since $\Phi$ is an isomorphism in degree zero, it restricts to an isomorphism of commutative algebras

$$
\operatorname{im}\left(\mathrm{H}^{2 *}\left(X_{2}, \overline{\mathbb{Q}}_{\ell}\right) \rightarrow \mathrm{IH}^{2 *}\left(X_{2}, \overline{\mathbb{Q}}_{\ell}\right)\right) \simeq B^{*}(f)_{\overline{\mathbb{Q}}_{\ell}} .
$$

The conclusion follows from the hard Lefschetz theorem for $L$ on $\mathrm{IH}^{2 *}\left(X_{2}, \overline{\mathbb{Q}}_{\ell}\right)$.

Theorem 6 will be deduced from the case when $f$ is the map $X_{\mathscr{A}} \rightarrow\left(\mathbb{P}^{1}\right)^{n}$. For each $i \in E$, let $f_{i}$ be the composition of $f$ with the $i$ th projection:

$$
f_{i}=\operatorname{proj}_{i} \circ f, \quad \operatorname{proj}_{i}:\left(\mathbb{P}^{1}\right)^{n} \longrightarrow \mathbb{P}^{1} .
$$

As in Proposition 9, for each $i \in E$ we write $\beta_{i}$ for the sum of $x_{\bar{F}}$ over all flats $\bar{F}$ of $\bar{M}$ that contain $i$ and do not contain 0 . As mentioned before, the blow-up construction of $X_{\mathscr{A}}$ shows that the cycle class map induces an isomorphism of commutative graded $\overline{\mathbb{Q}}_{\ell}$-algebras

$$
A^{*}\left(X_{\overline{\mathscr{A}}}\right) \otimes_{\mathbb{Q}} \overline{\mathbb{Q}}_{\ell} \simeq \mathrm{H}^{2 *}\left(X_{\mathscr{A}}, \overline{\mathbb{Q}}_{\ell}\right)
$$

Let $\Psi$ be the composition of isomorphisms

$$
\Psi: A^{*}(\overline{\mathrm{M}}) \otimes_{\mathbb{Q}} \overline{\mathbb{Q}}_{\ell} \simeq A^{*}\left(X_{\mathscr{A}}\right) \otimes_{\mathbb{Q}} \overline{\mathbb{Q}}_{\ell} \simeq \mathrm{H}^{2 *}\left(X_{\bar{A}}, \overline{\mathbb{Q}}_{\ell}\right)
$$

which maps $x_{\bar{F}}$ to the class of the strict transform in $X_{\bar{A}}$ of the exceptional divisor produced when blowing up the flat of $\overline{\mathscr{A}}$ in $\mathbb{P}^{r}$ corresponding to $\bar{F}$.

Proposition 13. The element $\Psi\left(\beta_{i}\right)$ is the pull-back of the class of a point in $\mathbb{P}^{1}$ under $f_{i}$. 
Proof. We factor $f$ into the composition

$$
X_{\mathscr{A}} \stackrel{j_{\mathscr{A}}}{\longrightarrow} X_{A_{n}} \stackrel{\pi_{2}}{\longrightarrow}\left(\mathbb{P}^{1}\right)^{n} .
$$

As noted before, the pull-back map associated with the inclusion $j_{\mathscr{A}}$ satisfies

$$
x_{\bar{I}} \longmapsto \begin{cases}x_{\bar{I}}, & \text { if } \bar{I} \text { is a flat of } \overline{\mathrm{M}}, \\ 0, & \text { if } \bar{I} \text { is a not flat of } \overline{\mathrm{M}} .\end{cases}
$$

Thus, it is enough to prove the claim when $X_{\mathscr{A}}=X_{A_{n}}$. This is the case when $\overline{\mathrm{M}}$ is the boolean matroid on $\bar{E}$, and the claim in this case was proved in Remark 11 at the level of Chow rings.

Since the cohomology ring of $\left(\mathbb{P}^{1}\right)^{n}$ is generated by the pull-backs under $f_{i}$, Propositions 9 and 13 together show that $\Psi$ induces an isomorphism between $B^{*}(\mathrm{M}) \otimes_{\mathbb{Q}} \overline{\mathbb{Q}}_{\ell}$ and $B^{*}(f)_{\overline{\mathbb{Q}}_{\ell}}$, which we denote by $\Psi^{\prime}$. More precisely, there is a commutative diagram

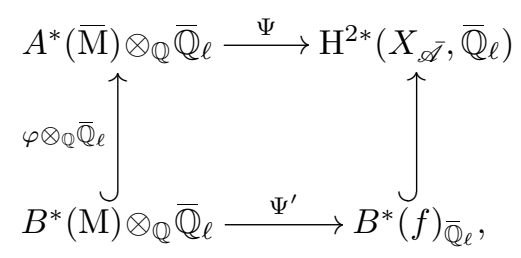

where $\varphi$ is the injective ring homomorphism of Proposition 9.

Proof of Theorem 6. It is enough to show that the multiplication map

$$
\begin{aligned}
B^{p}(\mathrm{M}) \otimes_{\mathbb{Q}} \overline{\mathbb{Q}}_{\ell} \longrightarrow B^{r-p}(\mathrm{M}) \otimes_{\mathbb{Q}} \overline{\mathbb{Q}}_{\ell}, \\
\xi \longmapsto L^{r-2 p} \xi
\end{aligned}
$$

is injective. Under the isomorphism $\Psi^{\prime}$, the statement to be proved translates to the conclusion of Proposition 12 when $f$ is the map $X_{\mathscr{A}} \rightarrow\left(\mathbb{P}^{1}\right)^{n}$.

With more work, we can show that the graded Möbius algebra of the matroid M is isomorphic to the cohomology ring of the variety $Y_{\mathscr{A}}$. Write $L_{i}$ for the first Chern class of the pull-back of $\mathcal{O}(1)$ under the composition

$$
Y_{\mathscr{A}} \longleftrightarrow\left(\mathbb{P}^{1}\right)^{n} \stackrel{\operatorname{proj}_{i}}{\longrightarrow} \mathbb{P}^{1}
$$

THEOREM 14. There is an isomorphism of commutative graded $\overline{\mathbb{Q}}_{\ell}$-algebras

$$
\begin{aligned}
B^{*}(\mathrm{M}) \otimes_{\mathbb{Q}} \overline{\mathbb{Q}}_{\ell} \stackrel{\simeq}{\longrightarrow} H^{2 *}\left(Y_{\mathscr{A}}, \overline{\mathbb{Q}}_{\ell}\right), \\
y_{i} \longmapsto L_{i} .
\end{aligned}
$$


In what follows, we write $z_{0}, z_{1}, \ldots, z_{n}$ for the homogeneous coordinates of $\mathbb{P}^{n}$, and write $\left(z_{1}, w_{1}\right), \ldots,\left(z_{n}, w_{n}\right)$ for the multi-homogeneous coordinates of $\left(\mathbb{P}^{1}\right)^{n}$.

Proof. Recall that $\mathrm{M}$ and $\overline{\mathrm{M}}$ share the same set of circuits. For every circuit $C$ of $\mathrm{M}$, there are non-zero constants $a_{c} \in \overline{\mathbb{F}}_{p}$, one for each element $c \in C$, such that

$$
\sum_{c \in C} a_{c} z_{c}=0 \quad \text { on the image of } i_{\mathscr{A}}: \mathbb{P}^{r} \longrightarrow \mathbb{P}^{n} .
$$

The collection $\left(a_{c}\right)_{c \in C}$ is uniquely determined by the circuit $C$, up to a common multiple.

A defining set of multi-homogeneous equations of $Y_{\mathscr{A}}$ is explicitly described by Ardila and Boocher in [AB, Theorem 1.3]:

$Y_{\mathscr{A}}=\left\{\left(\left(z_{1}, w_{1}\right), \ldots,\left(z_{n}, w_{n}\right)\right) \in\left(\mathbb{P}^{1}\right)^{n}: \sum_{c \in C} a_{c} z_{c}\left(\prod_{d \in C \backslash\{c\}} w_{d}\right)=0\right.$ for all circuits $C$ of $\left.\mathrm{M}\right\}$.

This shows that $Y_{\mathscr{A}}$ has an algebraic cell decomposition, in the sense of [BE, §3],

$$
Y_{\mathscr{A}}=\coprod_{F} \mathbb{A}^{\operatorname{rank}(F)},
$$

where the disjoint union is over all flats $F$ of $\mathrm{M}$, and $\mathbb{A}^{\operatorname{rank}(F)}$ is the intersection of $Y_{\mathscr{A}}$ with the affine space

$\mathbb{A}^{|F|}=\left\{\left(\left(z_{1}, w_{1}\right), \ldots,\left(z_{n}, w_{n}\right)\right) \in\left(\mathbb{P}^{1}\right)^{n}: w_{i}=0\right.$ if and only if $i$ is not in $\left.F\right\} \subseteq\left(\mathbb{P}^{1}\right)^{n}$

The existence of the cell decomposition has the following implications [BE, Theorem 3.1]:

(CD1) The natural map $\mathrm{H}^{2 *}\left(Y_{\mathscr{A}}, \overline{\mathbb{Q}}_{\ell}\right) \longrightarrow \mathrm{IH}^{2 *}\left(Y_{\mathscr{A}}, \overline{\mathbb{Q}}_{\ell}\right)$ is injective.

(CD2) The dimension of $\mathrm{H}^{2 k}\left(Y_{\mathscr{A}}, \overline{\mathbb{Q}}_{\ell}\right)$ is the number of $k$-dimensional cells in $Y_{\mathscr{A}}$ for all $k$.

All the odd-cohomology groups of $Y_{\mathscr{A}}$ are zero. When combined with the decomposition theorem for $X_{\mathscr{A}} \rightarrow Y_{\mathscr{A}}$, the statement (CD1) shows that the pull-back homomorphism in cohomology

$$
\mathrm{H}^{2 *}\left(Y_{\mathscr{A}}, \overline{\mathbb{Q}}_{\ell}\right) \longrightarrow \mathrm{H}^{2 *}\left(X_{\mathscr{A}}, \overline{\mathbb{Q}}_{\ell}\right)
$$

is injective. According to Proposition 13, the pull-back of $L_{i}$ in the cohomology of $X_{\mathscr{A}}$ is $\Psi\left(\beta_{i}\right)$, and hence the previous sentence implies that there is an injective graded ring homomorphism

$$
\begin{aligned}
B^{*}(f)_{\overline{\mathbb{Q}}_{\ell}} \longrightarrow \mathrm{H}^{2 *}\left(Y_{\mathscr{A}}, \overline{\mathbb{Q}}_{\ell}\right), \\
\Psi\left(\beta_{i}\right) \longmapsto L_{i}
\end{aligned}
$$


Composing with the isomorphism $\Psi^{\prime}$, we get the injective graded ring homomorphism

$$
\begin{aligned}
B^{*}(\mathrm{M}) \otimes_{\mathbb{Q}} \overline{\mathbb{Q}}_{\ell} & \longrightarrow \mathrm{H}^{2 *}\left(Y_{\mathscr{A}}, \overline{\mathbb{Q}}_{\ell}\right), \\
y_{i} & \longmapsto L_{i} .
\end{aligned}
$$

The statement $(\mathrm{CD} 2)$ shows that the source and the target are $\overline{\mathbb{Q}}_{\ell}$-vector spaces of the same dimension, which is the number of flats of M. Therefore, the map must be an isomorphism.

Remark 15. Let $\mathrm{M}$ be a simple matroid on $E=\{1, \ldots, n\}$ with rank $r \geqslant 2$. We write "deg" for the isomorphism

$$
\begin{aligned}
\operatorname{deg}: B^{r}(\mathrm{M}) & \longrightarrow \mathbb{Q}, \\
y_{E} & \longmapsto 1 .
\end{aligned}
$$

Let $\operatorname{HR}(\mathrm{M})$ be the symmetric $n \times n$ matrix with entries

$$
\operatorname{HR}(\mathrm{M})_{i j}= \begin{cases}0, & \text { if } i=j, \\ b_{i j}(\mathrm{M}), & \text { if } i \neq j,\end{cases}
$$

where $b_{i j}(\mathrm{M})$ is the number of bases of $\mathrm{M}$ containing $i$ and $j$. The matrix $\operatorname{HR}(\mathrm{M})$ represents the Hodge-Riemann form

$$
\begin{aligned}
B^{1}(\mathrm{M}) \times B^{1}(\mathrm{M}) & \longrightarrow \mathbb{Q}, \\
\left(\xi_{1}, \xi_{2}\right) & \longmapsto \operatorname{deg}\left(L^{r-2} \xi_{1} \xi_{2}\right),
\end{aligned}
$$

with respect to the standard basis $\left\{y_{1}, \ldots, y_{n}\right\}$. It can be shown that the matrix $\operatorname{HR}(\mathrm{M})$ has exactly one positive eigenvalue $[\mathrm{HW}]$.

Consider the restriction of $\mathrm{HR}(\mathrm{M})$ to the 3-dimensional subspace of $B^{1}(\mathrm{M})$ spanned by $y_{i}, y_{j}$ and $L$. The one-positive-eigenvalue condition says that the determinant of the resulting symmetric $3 \times 3$ matrix is non-negative, and this implies

$$
2>\frac{b(\mathrm{M}) b_{i j}(\mathrm{M})}{b_{i}(\mathrm{M}) b_{j}(\mathrm{M})}
$$

where $b(\mathrm{M})$ is the number of bases of $\mathrm{M}$ and $b_{i}(\mathrm{M})$ is the number of bases of $\mathrm{M}$ containing $i$. More detailed arguments will be given in $[\mathrm{HW}]$.

Question. How large can the ratio $b(\mathrm{M}) b_{i j}(\mathrm{M}) / b_{i}(\mathrm{M}) b_{j}(\mathrm{M})$ be?

For graphic matroids, the work of Kirchhoff on electric circuits shows that the ratio is bounded above by 1 , see [FM]. In other words, for a randomly chosen spanning tree of a graph, the presence of an edge can only make any other edge less likely. It was once conjectured that this is the case for all matroids, but Seymour and Welsh found an example with the ratio $\simeq 1.02[\mathrm{SW}]$. 


\section{Acknowledgements}

We thank Petter Brändén, Jeff Kahn, Satoshi Murai, Yasuhide Numata, Nick Proudfoot, Dave Wagner, and Geordie Williamson for helpful conversations. Special thanks go to two anonymous referees, who made very useful suggestions. This research started while Botong Wang was visiting Korea Institute for Advanced Study in summer 2016. We thank KIAS for excellent working conditions. June Huh was supported by a Clay Research Fellowship and NSF Grant DMS-1128155.

\section{References}

[AHK] Adiprasito, K., Huh, J. \& Katz, E., Hodge theory for combinatorial geometries. Preprint, 2015. arXiv:1511.02888 [math.CO].

[A] Aigner, M., Whitney numbers, in Combinatorial Geometries, Encyclopedia Math. Appl., 29, pp. 139-160. Cambridge Univ. Press, Cambridge, 1987.

[AB] Ardila, F. \& Boocher, A., The closure of a linear space in a product of lines. $J$. Algebraic Combin., 43 (2016), 199-235.

[BK] Basterfield, J.G. \& Kelly, L. M., A characterization of sets of $n$ points which determine $n$ hyperplanes. Proc. Cambridge Philos. Soc., 64 (1968), 585-588.

[BB] Batyrev, V. \& Blume, M., The functor of toric varieties associated with Weyl chambers and Losev-Manin moduli spaces. Tohoku Math. J., 63 (2011), 581-604.

[BBD] Bĕllinson, A. A., Bernstein, J. \& Deligne, P., Faisceaux pervers, in Analysis and Topology on Singular Spaces, I (Luminy, 1981), Astérisque, 100, pp. 5-171. Soc. Math. France, Paris, 1982.

[BL] Billera, L. J. \& Lee, C. W., Sufficiency of McMullen's conditions for $f$-vectors of simplicial polytopes. Bull. Amer. Math. Soc., 2 (1980), 181-185.

[B] BJörner, A., The unimodality conjecture for convex polytopes. Bull. Amer. Math. Soc., 4 (1981), 187-188.

[BE] BJörner, A. \& Ekedahl, T., On the shape of Bruhat intervals. Ann. of Math., 170 (2009), 799-817.

[dBE] De Bruijn, N. G. \& Erdős, P., On a combinatorial problem. Indagationes Math., 10 (1948), 421-423.

[C] Canfield, E. R., On a problem of Rota. Bull. Amer. Math. Soc., 84 (1978), 164.

[dCM] DE CATAldo, M. A. A. \& Migliorini, L., The decomposition theorem, perverse sheaves and the topology of algebraic maps. Bull. Amer. Math. Soc., 46 (2009), 535-633.

[dCP1] De Concini, C. \& Procesi, C., Wonderful models of subspace arrangements. Selecta Math., 1 (1995), 459-494.

[dCP2] - Hyperplane arrangements and holonomy equations. Selecta Math., 1 (1995), 495-535.

[DG] Dilworth, R. P. \& Greene, C., A counterexample to the generalization of Sperner's theorem. J. Combin. Theory Ser. A, 10 (1971), 18-21.

[DW1] Dowling, T. A. \& Wilson, R. M., The slimmest geometric lattices. Trans. Amer. Math. Soc., 196 (1974), 203-215.

[DW2] - Whitney number inequalities for geometric lattices. Proc. Amer. Math. Soc., 47 (1975), 504-512.

[EPW] Elias, B., Proudfoot, N. \& Wakefield, M., The Kazhdan-Lusztig polynomial of a matroid. Adv. Math., 299 (2016), 36-70. 
[FM] Feder, T. \& Minail, M., Balanced matroids, in Proc. of the 24th ACM Symposium on Theory of Computing, pp. 26-38. ACM, New York, NY, 1992.

[FY] Feichtner, E. M. \& Yuzvinsky, S., Chow rings of toric varieties defined by atomic lattices. Invent. Math., 155 (2004), 515-536.

[F1] Fulton, W., Introduction to Toric Varieties. Ann. of Math. Stud., 131. Princeton Univ. Press, Princeton, NJ, 1993.

[F2] - Young Tableaux. London Math. Soc. Stud. Texts, 35. Cambridge Univ. Press, Cambridge, 1997.

[F3] - Intersection Theory. Ergebnisse der Mathematik und ihrer Grenzgebiete, 2. Springer, Berlin-Heidelberg, 1998.

[G] Greene, C., A rank inequality for finite geometric lattices. J. Combinatorial Theory, 9 (1970), 357-364.

$\left[\mathrm{HM}^{+}\right]$Harima, T., Maeno, T., Morita, H., Numata, Y., Wachi, A. \& Watanabe, J., The Lefschetz Properties. Lecture Notes in Math., 2080. Springer, Berlin-Heidelberg, 2013.

[Ha] Hausel, T., Quaternionic geometry of matroids. Cent. Eur. J. Math., 3 (2005), 26-38.

[He] Heron, A. P., A property of the hyperplanes of a matroid and an extension of Dilworth's theorem. J. Math. Anal. Appl., 42 (1973), 119-131.

[HW] Huh, J. \& WAng, B., Mason's conjecture and the Hodge-Riemann relations for matroids. In preparation.

[Ke] KeEL, S., Intersection theory of moduli space of stable $n$-pointed curves of genus zero. Trans. Amer. Math. Soc., 330 (1992), 545-574.

[Ku1] Kung, J. P. S., The Radon transforms of a combinatorial geometry. I. J. Combin. Theory Ser. A, 26 (1979), 97-102.

[Ku2] - Radon transforms in combinatorics and lattice theory, in Combinatorics and Ordered Sets (Arcata, CA, 1985), Contemp. Math., 57, pp. 33-74. Amer. Math. Soc., Providence, RI, 1986.

[Ku3] - The Radon transforms of a combinatorial geometry. II. Partition lattices. Adv. Math., 101 (1993), 114-132.

[Ku4] - On the lines-planes inequality for matroids. J. Combin. Theory Ser. A, 91 (2000), 363-368.

[KRY] Kung, J. P. S., Rota, G. C. \& Yan, C. H., Combinatorics: The Rota Way. Cambridge Mathematical Library. Cambridge Univ. Press, Cambridge, 2009.

[MN] Maeno, T. \& Numata, Y., Sperner property, matroids and finite-dimensional Gorenstein algebras, in Tropical Geometry and Integrable Systems, Contemp. Math., 580, pp. 73-84. Amer. Math. Soc., Providence, RI, 2012.

[Ma] Mason, J.H., Matroids: unimodal conjectures and Motzkin's theorem, in Combinatorics (Combinatorial Math., Oxford, 1972), pp. 207-220. Inst. Math. Appl., Southend-on-Sea, 1972.

[Mo1] Motzkin, T. Beiträge zur Theorie der linearen Ungleichungen, 1936. Dissertation Thesis, University of Basel, Jerusalem.

[Mo2] - The lines and planes connecting the points of a finite set. Trans. Amer. Math. Soc., 70 (1951), 451-464.

[O] OxLey, J., Matroid Theory. Oxford Graduate Texts in Mathematics, 21. Oxford Univ. Press, Oxford, 2011.

[PS] Proudfoot, N. \& Speyer, D., A broken circuit ring. Beiträge Algebra Geom., 47 (2006), 161-166.

[R] Rota, G.-C., Combinatorial theory, old and new, in Actes du Congrès International des Mathématiciens (Nice, 1970), Tome 3, pp. 229-233. Gauthier-Villars, Paris, 1971. 
[RH] Rota, G.-C. \& Harper, L.H., Matching theory, an introduction, in Advances in Probability and Related Topics, Vol. 1, pp. 169-215. Dekker, New York, 1971.

[SW] Seymour, P. D. \& Welsh, D. J. A., Combinatorial applications of an inequality from statistical mechanics, 77, pp. 485-495. 1975.

[So] Solomon, L., The Burnside algebra of a finite group. J. Combinatorial Theory, 2 (1967), 603-615.

[Stl1] Stanley, R. P., Combinatorics and Commutative Algebra. Progress in Mathematics, 41. Birkhäuser, Boston, MA, 1996.

[St12] - Enumerative Combinatorics. Vol. 1, second edition. Cambridge Studies in Advanced Mathematics, 49. Cambridge Univ., Cambridge, 2012.

[St13] - Algebraic Combinatorics. Undergraduate Texts in Mathematics. Springer, New York, 2013.

[Stt] Stanton, D., Unimodality and Young's lattice. J. Combin. Theory Ser. A, 54 (1990), $41-53$

[W] Welsh, D. J. A., Matroid Theory. London Math. Soc. Monogr. Ser., 8. Academic Press, London-New York, 1976.

[dW1] DE Witte, P., Combinatorial properties of finite linear spaces. I. Bull. Soc. Math. Belg., 18 (1966), 133-141.

[dW2] - Combinatorial properties of finite linear spaces. ii. Bull. Soc. Math. Belg., 27 (1975), $115-155$

[Z] Ziegler, G. M., Lectures on Polytopes. Graduate Texts in Mathematics, 152. Springer, New York, 1995.

JunE HuH

Institute for Advanced Study

Fuld Hall

1 Einstein Drive

Princeton, NJ

U.S.A.

huh@princeton.edu

and

Korea Institute for Advanced Study

85 Hoegiro, Dongdaemun-gu

Seoul 130-722

Korea

Received October 9, 2016

Received in revised form January 30, 2017
Botong WANG

University of Wisconsin-Madison

Van Vleck Hall

480 Lincoln Drive

Madison, WI

U.S.A.

bwang274@wisc.edu 